\title{
Tumor Therapeutic Response and Vessel Tortuosity: Preliminary Report in Metastatic Breast Cancer
}

\author{
Elizabeth Bullitt ${ }^{1}$, Nancy U. Lin ${ }^{2}$, Matthew G. Ewend ${ }^{1}$, Donglin Zeng ${ }^{1}$, \\ Eric P. Winer ${ }^{2}$, Lisa A. Carey ${ }^{1}$, and J. Keith Smith ${ }^{1}$ \\ ${ }^{1}$ CASILab, CB \# 7062, University of North Carolina, Chapel Hill, NC 27599, USA \\ \{bullitt, ewend, jksmith, carey\} amed.unc.edu, \\ dzeng@bios.unc.edu \\ http: / / casilab.med.unc.edu / \\ ${ }^{2}$ Dana-Farber/Harvard Cancer Center, Boston, MA 02115, USA \\ \{Nancy_Lin, Eric_Winer\}@dfci.harvard.edu
}

\begin{abstract}
No current non-invasive method is capable of assessing the efficacy of brain tumor therapy early during treatment. We outline an approach that evaluates tumor activity via statistical analysis of vessel shape using vessels segmented from MRA. This report is the first to describe the changes in vessel shape that occur during treatment of metastatic brain tumors as assessed by sequential MRA. In this preliminary study of 16 patients undergoing treatment for metastatic breast cancer we conclude that vessel shape may predict tumor response several months in advance of traditional methods.
\end{abstract}

\section{Introduction}

Effective monitoring of brain tumor therapy poses a major clinical problem. If a tumor previously sensitive to a drug later becomes resistant, the therapeutic regimen should be changed rapidly. Unfortunately, there is presently no reliable, noninvasive means of monitoring therapeutic efficacy. Biopsy often provides an answer but is too invasive to be performed frequently. Change in neurological examination is helpful but often occurs late in the clinical course and is an insensitive measure of tumor growth.

The current standard of practice is to monitor tumor treatment by performing magnetic resonance (MR) scans at regular intervals and to assess tumor growth by measuring the size of the tumor on gadolinium-enhanced images in one, two, or three dimensions $[1,2,3]$. Such measurements can then be compared to similar measurements made from a later scan. Regardless of the relative merits of each of these approaches (1D estimates are more crude than 2D, and 2D are more crude than $3 \mathrm{D}$ ), all three assessments give only anatomical information about what the tumor is doing "now", cannot predict what the tumor will do "next", and provide basically similar information [4].

A reliable method of monitoring tumor metabolic or physiologic activity would be of higher value, since it could inform the clinician what the tumor is about to do "next". As a result, many techniques are under development, including positron emission tomography, MR spectroscopy, and perfusion imaging. Reviews are provided by Benard [5] and Law [6]. None of these techniques have yet proven clinically reliable. 
This report provides an initial evaluation of a new method of assessing tumor treatment response over time. The approach employs a quantitative, statistical measure of vessel tortuosity using vessels segmented from high-resolution MR angiograms (MRA). Subjects were those with breast cancer metastatic to brain and enrolled in a multi-center trial aimed at evaluating the response of brain mestastases to an experimental drug. Tumor status was evaluated for each patient by vessel tortuosity measurements, by three-dimensional calculation of tumor volume, and by clinical criteria, which included uni-dimensional assessment of tumor size on Gadolinium-enhanced MR images by RECIST criteria [1]. Our hypothesis was that sequential measures of vessel shape would correctly predict tumor response in advance of both clinical criteria and the volumetric calculation of tumor size made from MR.

\section{Methods}

All subjects were enrolled in a five-center drug trial aimed at treating breast cancer metastatic to brain. MR scans of the head were obtained prior to initiation of therapy and every two months thereafter, with additional scans sometimes acquired for clinical reasons. Patients were withdrawn from the drug study for progressive intracranial disease if they met RECIST criteria for tumor enlargement [1] - a one-dimensional assessment of tumor size made from gadolinium enhanced images. Neither vessel shape measures nor 3D tumor volumetric assessments were used as the basis for clinical decision. The current imaging study describes results in the 16 patients who, as of January 1 2006, had undergone at least two good-quality MRA examinations, whose images were available for analysis, and who had been withdrawn from the drug study for progressive intracranial disease. Some of these subjects exhibited initial response to therapy. Subjects still undergoing treatment and subjects withdrawn from the drug study for progressive extracranial disease were excluded from the current report as such patients had undefined endpoints for intracranial disease.

All patients in the current study received sequential MRA and T1-gadolinium enhanced images obtained on various 1.5T Siemens, GE, and Phillips MR scanners. We required standardized, time-of-flight, MRA images from which to perform a statistical analysis of vessel shape. We therefore made cross-institutional requirements for MRA acquisitions that required repeated scanning of the same patient on the same machine, coverage of the entire head, and voxel size of $0.5 \times 0.5 \times 0.8 \mathrm{~mm}^{3}$. Any MRA that failed to meet these criteria was excluded. The protocols used for T1-gadolinium enhanced imaging were more variable. We strongly encouraged all institutions to acquire $\mathrm{T} 1$ images at $3 \mathrm{~mm}$ interslice spacing or less, but did not exclude a patient from analysis if these recommendations were not followed.

Vessels were segmented from each MRA using a method that, proceeding from a seed point, automatically defined each vessel as a set of regularly spaced skeleton points with an associated radius at each point [7]. A second, semi-automatic program was then used to define vessel trees and to exclude veins and the external circulation [8]. The segmentation process defined each vessel as an ordered, regularly spaced set of $\mathrm{x}, \mathrm{y}, \mathrm{z}$ skeleton points with an associated radius at each point. Vessel segmentation and tree formation required approximately 30 minutes. 
All tumors of $1 \mathrm{~cm}^{3}$ or more were defined from T1-gadolinium enhanced images using a program that defined tumors via polygon drawing and filling on orthogonal cuts through an image volume. Tumor volume was automatically calculated as the number of labeled voxels multiplied by voxel size, with results expressed in cubic $\mathrm{cm}$. For the current study, a meaningful increase (decrease) in tumor volume was defined as a volumetric increase (decrease) from baseline values of at least $20 \%$ with a concomitant change in volume of at least $0.5 \mathrm{~cm}^{3}$.

We had initially planned to analyze vessel shapes on a regional basis, viewing each defined tumor as an independent entity. So many lesions were present, however, that this approach proved impractical. The entire brain was therefore taken as the region of interest, and results are here reported for each patient's entire intracranial circulation regardless of the number of metastatic tumors, their locations, or their volumes.

Although many vessel shape measures were calculated for each subject, the current report analyzes only the "malignancy probability" (MP). The MP equation was derived from an earlier, blinded study of benign and malignant tumors. This earlier study concluded via discriminant analysis of multiple vessel shape parameters that only a combination of two tortuosity metrics appeared effective in generically separating benign from malignant disease [9]. One of these metrics, the "Sum of Angles Metric" (SOAM), sums curvature along a space curve and normalizes by vessel length [10]. The second metric, the "Inflection Count Metric" (ICM), multiplies the total path length of a space curve by the number of inflection points and divides by the distance between endpoints [10]. The SOAM is effective in flagging highfrequency, low amplitude curves. The ICM is effective in flagging large amplitude curves that frequently change direction. Each metric describes a different form of tortuosity. The combination of the two metrics quantitatively describes the vessel shape abnormality typically associated with cancer, aptly described by Baish as "many smaller bends upon each larger bend" [11], with SOAM quantifying the small bends and ICM the larger ones. More information about tortuosity metrics is given in [10] and derivation of the combinatorial equation is described in [9]. What the minimum clinically meaningful change in the MP should be is unknown. For the current study, we defined in advance that evidence of tumor response required a drop in MP by $>=20$ from baseline value.

MP calculation requires normalization of each tortuosity value by the means and standard deviations of healthy vessel values via z-scoring [9]. Data from 34 healthy subjects, ranging in age from 18-72 and including subjects of both sexes, were used for this normalization.

\section{Results}

Tumor presentation as visualized by T1-gadolinium enhanced images differed from subject to subject. The majority of patients exhibited multiple tiny metastases as well as 1-4 lesions of $1 \mathrm{~cm}^{3}$ or more. Vessel abnormalities were widespread throughout the head, and consisted of the "many smaller bends upon each larger bend" [11] typical of cancer-associated vasculature. Figure 1 illustrates these typical vessel shape abnormalities as well as their improvement during successful treatment. 

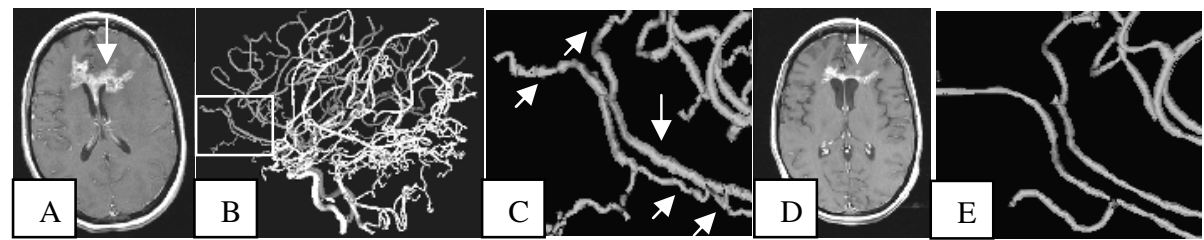

Fig. 1. Improvement of vessel tortuosity abnormalities during successful treatment. A: Axial slice of a T1-GAD baseline scan showing a large tumor (arrow). B: Lateral view of the vessels segmented from the baseline MRA. Rectangle outlines the region magnified in C. C: Tortuosity abnormalities involve both smaller branches and the large frontopolar arteries (arrows). D: The tumor has regressed at month 2 of treatment (arrow). E: At month 2 small, abnormal vessel branches have largely disappeared and there is normalization of the larger vessels' shapes.

Table 1 provides summary results of the time in months for clinical criteria, volumetric measurements, and vessel shape analysis to indicate tumor progression from the time of the baseline scan. The table is divided into five groups, each indicative of a different set of results.

Group 1 consists of 4 patients who exhibited clear-cut evidence of tumor growth by both clinical criteria and volumetric measurement at 2 months. Tumor growth was often explosive, with volumetric increases of as much as $200 \%$ and $5 \mathrm{~cm}^{3}$. All patients exhibited a high MP by vessel analysis both at baseline and at month 2 . Although vessel analysis was consistent with other measures of tumor progression, it did not provide added information. By any criterion, this group of patients failed treatment.

Group 2 consists of 4 patients who were removed from the drug trial at month 4 or 6 , but in whom both volumetric measurement and vessel analysis would have correctly indicated therapeutic failure at month 2. All 4 patients exhibited tumor growth by more than $20 \%$ volume and 0.5 cubic centimeters at month 2 with continued growth thereafter, and none exhibited improvement in MP values at any time point. In these 4 cases volumetrics and vessel analysis performed equally well, and both performed better than clinical RECIST criteria [1] in flagging tumor progression.

Group 3 consists of an important group of 5 patients who demonstrated stable disease or tumor remission volumetrically at month 2 , but in whom vessel analysis indicated continued high malignancy at month 2 . Any volumetric improvement was transient in these subjects. All patients exhibited tumor growth volumetrically over baseline at month 4 , with monotonic increase in tumor volume thereafter. In these 5 cases, vessel analysis correctly predicted tumor activity 2 to 4 months in advance of all methods based upon gadolinium-enhanced imaging. Figure 2 (left) provides an example.

Group 4 consisted of one patient who had multiple tiny lesions, all too small to analyze meaningfully either volumetrically or by RECIST [1] criteria. She was withdrawn from the drug study at month 4 for clinical worsening; vessel analysis correctly indicated high ongoing tumor activity at month 2 .

Group 5 contains the only two subjects who showed significant improvement in vessel malignancy probability at month 2 . It is of note that these two subjects were also the only ones to show sustained improvement or stability in tumor volume. Subject 003 demonstrated progressive, dramatic reduction in lesion volume until month 4 , 
Table 1. Time in months at which tumor progression was noted by clinical criteria (CLIN), volumetrics (VOL), and by vessel malignancy probability (MP). Subjects are classified into 5 response groups (column 1), with the patient identification number given in column 2. Column 5 provides comments, with "CM" indicating carcinomatous meningitis and "Volume smaller?" indicating a volumetric decrease insufficient to meet formal criteria for volumetric reduction.

\begin{tabular}{|c|c|c|c|c|c|}
\hline Group & Study ID & CLIN & VOL & MP & COMMENT \\
\hline 1 & 007 & 2 & 2 & 2 & Failed by all criteria \\
\hline 1 & 009 & 2 & 2 & 2 & Failed by all criteria \\
\hline 1 & 016 & 2 & 2 & 2 & Failed by all criteria \\
\hline 1 & 022 & 2 & 2 & 2 & Failed by all criteria \\
\hline 2 & 006 & 4 & 2 & 2 & Monotonic 3D tumor growth \\
\hline 2 & 010 & 6 & 2 & 2 & Monotonic 3D tumor growth \\
\hline 2 & 026 & 4 & 2 & 2 & Monotonic 3D tumor growth \\
\hline 2 & 029 & 4 & 2 & 2 & Monotonic 3D tumor growth \\
\hline 3 & 008 & 4 & 4 & 2 & Slow, monotonic tumor growth \\
\hline 3 & 012 & 7 & 6 & 2 & Volume smaller? month 2 only \\
\hline 3 & 015 & 6 & 4 & 2 & Volume smaller month 2 only \\
\hline 3 & 023 & 4 & 4 & 2 & Volume smaller? month 2 only \\
\hline 3 & 027 & 3 & 3 & 2 & Volume smaller? month 2 only \\
\hline 4 & 018 & 4 & $>4$ & 2 & Only tiny lesions \\
\hline 5 & 003 & 11 & $>10$ & 4 & Volume smaller; new CM \\
\hline 5 & 013 & 6 & $>6$ & 6 & Lesion stable after month 2 \\
\hline
\end{tabular}
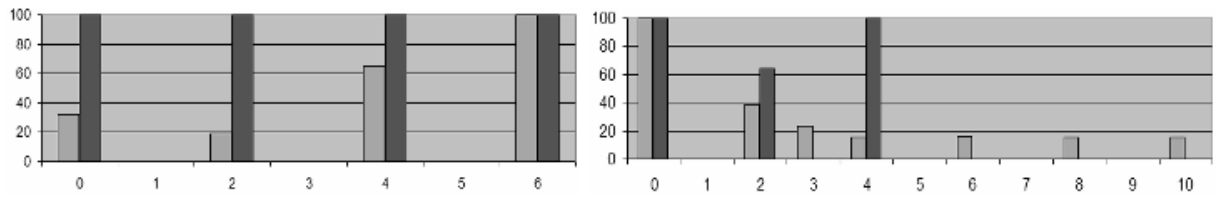

Fig. 2. Two examples of tumor volume reduction in which vessel MP values correctly presaged tumor activity. In both graphs, the $\mathrm{x}$ axis gives time in months (time 0 is the baseline scan prior to treatment). The y axis gives a percentage (0-100\%) representing both the malignancy probability (black bar) and the tumor percent volume (grey bar) in which the numerator is the tumor volume and the denominator the maximum tumor volume recorded for that patient; the tumor volume is $100 \%$ at the time point it was largest during the study. Left: Case 015 . The tumor regressed significantly at month 2 but subsequently enlarged rapidly; vessel MP correctly flagged the tumor as of high (100\%) MP at month 2, presaging worsening during the next cycle. Right: Case 003. The tumor volume progressively regressed between months 0 and 4 with vessel MP indicating a drop in activity from $100 \%$ to $64 \%$ at month 2, correctly presaging future improvement during the next 2-month cycle. MP returned to $100 \%$ at month 4 at which time the tumor stopped regressing; the subject also developed carcinomatous meningitis. No MRA data were available after month 4.

with subsequent stability of lesion volume through the time she was withdrawn from study at month 11 for carcinomatous meningitis. Her vessel malignancy probability improved significantly at month 2 (Figures 1,2), correctly presaging ongoing 
improvement, but reverted to her baseline $100 \%$ level at month 4 . It is unknown at which time point she began to develop microscopic leptomeningeal metastases. Neither volumetric nor RECIST measurements were able to delineate her impending treatment failure since these measurements were based upon the size of her solid tumor (Figure 3) rather than upon overall metabolic/physiologic activity.
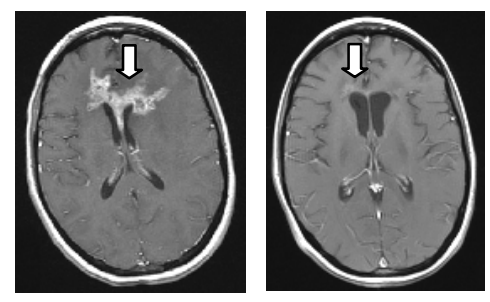

Fig. 3. Baseline $\mathrm{T} 1$ gadolinium-enhanced scan (left) and at 10 months (right) in a patient who exhibited an $88 \%$ reduction in lesion volume but developed carcinomatous meningitis

Case 013 is more difficult to interpret because she is missing a critical time point. Her tumor showed a volumetric increase between baseline and month 2 but subsequent volumetric stability through month 6 , at which time she was (perhaps incorrectly) withdrawn from the drug study for meeting RECIST criteria. Her vessel MP had dropped to lower levels at months 2 and 4 but reverted to her baseline level at month 6 . We believe that her tumor would have exhibited significant growth at month 8 on the basis of her vessel MP values, but image data from that time point are not available.

\section{Discussion}

Early assessment of tumor treatment response would be of high benefit to clinical therapeutic management. Current methods of evaluating drug therapy are inaccurate, delay diagnosis, and may even reach the wrong conclusion [16; Fig 3]. Commonly employed RECIST criteria involve estimating a change in a three-dimensional tumor volume using a single, noisy, 1-dimensional measurement, and even volumetric determination provides only anatomical information based upon a single imaging modality. Fundamentally, any method based solely upon analysis of gadolinium enhanced images will only be able to compare what the tumor size appears to be "now" to its size in the past, with additional confounding factors produced by successful treatment resulting in tumor necrosis, which can also induce gadolinium enhancement.

The use of sequential vessel shape analyses to measure cancer treatment response offers several theoretical advantages. First, abnormal vessel tortuosity provides an early flag of cancer activity. In experimental animal models, abnormal vessel tortuosity appears within 24 hours of subcutaneous injection of cancer cells [12]. Indeed, this abnormality appears when the tumor burden is only 60-80 cells and occurs earlier than neoangiogenic sprouting [12]! Second, the typical vessel shape abnormality of "many smaller bends upon each larger bend" [11] appears across anatomical regions, 
across cancer types, and even across species. The same malignancy probability equation can thus be used to flag cancer activity in different locations [13]. Third, vessel shape abnormalities extend far beyond tumor confines and involve initially healthy vessels coursing in the tumor vicinity $[9,12]$, thus making it feasible to perceive such vessels using high-resolution MRA even though MRA cannot depict capillaries. Fourth, the vessel shape abnormalities associated with active cancer appear to be independent of tissue vascularity as measured by perfusion imaging [14]. This capacity provides a significant strength when assessing hypovascular malignant or hypervascular benign tumors [9]. Finally and importantly, histological analyses in animal models have described rapid normalization of vessel shape abnormalities during successful treatment $[12,15]$. For a variety of reasons, the quantitative assessment of vessel shape thus appears to be an attractive approach for monitoring tumor treatment response.

The current study is the first to describe vessel shape changes in a set of human cancer patients imaged serially by MR during treatment. In these patients, vessel shape analyses provided earlier information about tumor progression than clinical RECIST criteria in 11/16 cases and than volumetric assessment in 7/16 cases. In these subsets of patients, tumor progression could have been correctly identified 2-6 months earlier by vessel analysis than by the standard methods used to assess tumor growth. In the remaining cases, vessel analysis performed equally well.

The ability of vessel analysis to correctly predict tumor regression in response to treatment is more difficult to define from this study. Only two subjects exhibited long-term volumetric stability or decrease in lesion size. It is important to note that the only two subjects to exhibit volumetric tumor suppression for 4 months or more were also the only two in which vessel malignancy probability exhibited reduction at month 2. However, meaningful statistical analysis is impossible with this small number of responders. The current study should therefore be interpreted as providing interesting preliminary results only. Definitive conclusions will require a much larger number of subjects responsive to therapy.

An important question is that of the time over which it takes vessel shapes to normalize during successful treatment and to revert to malignant patterns during tumor recurrence. Although histological studies in animals indicate that such pattern changes occur rapidly $[12,15]$, MR cannot discriminate the tiny vessels likely to be earliest affected. The time required for tortuosity changes to develop in the larger vessels delineable from MR is unknown. On the basis of the current study which studies a particular tumor type, a particular form of therapy, a small number of patients, and with MR scans performed at 2 month intervals, it appears that vessel shape changes indicative of response occur within 2 months and that vessel shape changes can predict what a tumor is about to do during at least the next 2 month period. Shorter scanning intervals might allow even more rapid determination of future tumor behaviour.

\section{Acknowledgments}

Supported by R01EB000219(NIBIB), P50CA58185-AV-55P2(Avon), P30CA1608629S1(NCI), P30CA58223 (NCI), and M01RR00046 (NIH). 


\section{References}

1. Therasse P, Arbuck SG, Eisenhauer EA, et al: New guidelines to evaluate the response to treatment in solid tumors: European Organization for Research and Treatment of Cancer, National Cancer Institute of the United States, National Cancer Institute of Canada. J Natl Cancer Inst 92 (2000) 205-216.

2. Macdonald DR, Cascino TL, Schold SC Jr, Cairncross JG.: Response criteria for phase II studies of supratentorial malignant glioma. J Clin Oncol 8 (1990) 1277-1280.

3. Dempsey MF, Condon BR, Hadley DM: Measurement of tumor "size" in recurrent malignant glioma: 1D, 2D, or 3D? AJNR 26 (2005) 770-776.

4. Shah GD, Kesari S, Xu R, Batchelor TT, et al:Comparison of linear and volumetric criteria in assessing tumor response in adult high-grade gliomas. Neuro-Oncology 2005. Available at http://puck.ingentaconnect.com/vl=6507458/cl=14/nw=1/rpsv/cw/www/dup $/ 15228517 /$ previews/contp1-1.htm

5. Benard F, Romsa J, Hustinx R: Imaging gliomas with positron emission tomography and single-photon emission computed tomography. Seminars in Nuclear Medicine 33 (2003) 148-162.

6. Law M, Yang S, Wang H, Babb JS, Johnson G, Cha S, Knopp EA, Zagzag D: Glioma Grading:Sensitivity, specificity, and predictive values of perfusion MR imaging and proton MR spectroscopic imaging compared with conventional MR imaging. AJNR 24 (2003) 1989-1998.

7. Aylward, S.R., Bullitt, E.: Initialization, noise, singularities and scale in height ridge traversal for tubular object centerline extraction. IEEE-TMI 21 (2002) 61-75.

8. Bullitt, E., Aylward, S., Smith, K., Mukherji, S., Jiroutek, M., Muller, K.: Symbolic Description of Intracerebral Vessels Segmented from MRA and Evaluation by Comparison with X-Ray Angiograms. Medical Image Analysis 5 (2001) 157-169.

9. Bullitt E, Zeng D, Gerig G, Aylward S, Joshi S, Smith JK, Lin W, Ewend MG. Vessel tortuosity and brain tumor malignancy: A blinded study. Academic Radiology 12 (2005) 1232-1240.

10. Bullitt E, Gerig G, Pizer S, Aylward SR. Measuring tortuosity of the intracerebral vasculature from MRA images. IEEE-TMI 22 (2003) 1163-1171.

11. Baish JS, Jain RK. Fractals and cancer. Cancer Research 60 (2000) 3683-3688.

12. Li CH, Shan S, Huang Q, Braun R, Lanzen J, Hu K, Lin P, Dewhirst M, Initial stages of tumor cell-induced angiogenesis: evaluation via skin window chambers in rodent models. J Natl Cancer Inst 92 (2000) 143-147.

13. Bullitt E, Wolthusen A, Brubaker L, Lin W, Zeng D, Van Dyke T. Malignancy-associated vessel tortuosity: A computer-assisted, MRA study of choroid plexus carcinoma in genetically engineered mice. AJNR 27 (2006) 612-619

14. Parikh A, Smith JK, Ewend MG, Bullitt E. Correlation of MR perfusion imaging and vessel tortuosity parameters in assessment of intracranial neoplasms. Technology in Cancer Research and Treatment 3 (2004) 585-590.

15. Jain RK. Normalizing tumor vasculature with anti-angiogenic therapy: a new paradigm for combination therapy. Nature Medicine 7 (2001) 987-98. 